\title{
A parametric study of strength reduction factors for elasto-plastic oscillators
}

\author{
DEBASIS KARMAKAR and VINAY K GUPTA
}

Dept. of Civil Engineering, Indian Institute of Technology, Kanpur 208 016, India

e-mail: vinaykg@iitk.ac.in

\begin{abstract}
Strength reduction factors (SRFs) continue to play a key role in obtaining design forces from elastic design spectra (via response modification factors) in ductility-based earthquake-resistant design. Despite several years of sustained research efforts, it has not been conclusively shown how SRF for a given singledegree-of-freedom structural system depends on various source and site parameters. A parametric study is carried out here for the explicit dependence of SRF spectrum (describing variation of SRF with system period for a given ductility demand) on strong motion duration, earthquake magnitude, geological site conditions, and epicentral distance in case of (non-degrading) elasto-plastic oscillators. For this, scaled response spectra are considered for different combinations of earthquake magnitude, site conditions and epicentral distance, and SRF spectra are generated from 1274 accelerograms recorded in western USA after making those compatible with each of these spectra. It is shown that there is no clear and significant dependence of SRF spectrum on strong motion duration. Further, the parametric dependence on earthquake magnitude, site conditions, and epicentral distance broadly conforms to the trends reported by earlier investigations. In particular, this study confirms that the dependence of SRF spectra on earthquake magnitude should not be ignored.
\end{abstract}

Keywords. Strength reduction factors; elastic design spectra; ductility-based design.

\section{Introduction}

Due to economic considerations, earthquake-resistant design of structures involves the provision of design lateral strengths at much lower levels than those required for the elastic behaviour during the design ground motions. It is assumed that the structures will undergo inelastic deformations, to the extent determined by the ductility demand ratio, without collapse during these motions. Here, ductility demand (ratio) is the ratio of the maximum inelastic displacement of the structure to its yield displacement during the ground motion. The factor used to scale down the lateral strength demand is called as the response modification factor, which is product of (i) strength reduction factor (SRF), (ii) structural overstrength factor, and

This paper has been contributed in honour of Professor R N Iyengar, Indian Institute of Science, on the occasion of his formal retirement. 
(iii) redundancy factor (ATC 1995). SRF accounts for the nonlinear characteristics and ductility capacity of the structure, and hence is the most important component of the response modification factor. It is defined as the ratio of elastic strength demand of a single-degree-offreedom (SDOF) oscillator to the inelastic strength demand such that the ductility demand is limited to a specified value. This factor is also responsible for much of the parametric dependence of response modification factor on various structural and ground motion parameters.

Parametric dependence of SRF has been the topic of several investigations. Newmark \& Hall (1973) were the first to study this factor and to describe the dependence of this factor on the ductility (demand) ratio and initial period of the SDOF oscillator in an approximate manner. Riddell \& Newmark (1979) showed that it may be conservative to use an elastoplastic oscillator for computing SRF. Elghadamsi \& Mohraz (1987) found that for a given ductility (demand) ratio, reduction in elastic forces may be slightly more for a structure on rock than for a structure on an alluvium site. Peng et al (1988) showed that deamplification factors (reciprocals of SRFs) for the short and medium-period oscillators may be higher (implying smaller reductions in elastic forces) for the motions of longer durations. Krawinkler \& Nassar (1990) showed that SRFs (for oscillators of different periods) may be independent of epicentral distance while those are slightly sensitive to the type of hysteretic model chosen. Krawinkler \& Rahnama (1992) showed that in the case of soft soil sites, local site conditions may have significant effects on SRFs. Miranda (1993) found that earthquake magnitude has negligible effects on SRFs.

Most of the above studies were based on mean SRFs computed for different suites of ground motions such that the effects of only one or two ground motion parameters were studied at a time. Also, due to the inherent size limitations of the data-sets used, those did not properly isolate the effects of the governing parameter being considered from the effects of the other parameters. Tiwari \& Gupta (2000) attempted to address this limitation through scaling models for SRFs in terms of earthquake magnitude, strong motion duration, predominant period of ground motion, geological site conditions and ductility demand. They showed that the previously assumed insensitivity of earthquake magnitude and strong motion duration may not always hold good, and that the effects of these parameters may be dependent on the initial period of the oscillator. Chakraborti \& Gupta (2005) followed a similar approach and showed that degradation characteristics may be important only for high ductility demands.

It is clear from the above that SRFs may depend on different ground motion parameters like strong motion duration, earthquake magnitude and site conditions. It has not been however brought out clearly how exactly and to what extent do those depend on each of these parameters, independently of the other parameters. This is partly due to the fact that conflicting conclusions have been reported by different investigators and partly because none of the investigators explicitly studied the role of any of these parameters while keeping the other parameters unchanged. The models proposed by Tiwari \& Gupta (2000) addressed this situation, but their conclusions need to be examined more directly.

A parametric study of SRF spectrum (showing variation of SRF with the initial timeperiod of the oscillator for a given ductility demand) is carried out here with respect to the following parameters: strong-motion duration, earthquake magnitude, site conditions and epicentral distance. This study is based on the use of elasto-plastic oscillators with three levels of ductilities, and on a collection of 1274 accelerograms recorded during the 106 earthquake events in the western USA region from 1931 to 1984 (see Lee \& Trifunac 1987, for further details) and during the Northridge earthquake, 1994. Further, ensembles of synthetic accelerograms are obtained by modifying the recorded accelerograms to match different scaled response spectra, as proposed by Trifunac \& Lee (1985) for a given set of earthquake 
magnitude, site conditions, epicentral distance, and focal depth. Since the accelerograms considered here have different nonstationary characteristics and strong motion durations, the parametric study first focusses on strong motion duration, in order to see the extent to which this parameter may affect the SRF spectrum. The parametric studies for earthquake magnitude, site conditions, and epicentral distance are carried out by considering seven different pseudo spectral acceleration (PSA) spectra representing different sets of these parameters.

\section{Database of SRF spectra}

SRF spectrum, $R_{\mu}(T)$ describes the variation of SRF with the initial period, $T$, of a nonlinear SDOF oscillator. SRF is defined as the ratio of elastic strength demand to inelastic strength demand such that the displacement ductility ratio is limited to a maximum value of $\mu$, where ductility ratio, $\mu$, is the ratio of the maximum inelastic displacement of the oscillator to its yield displacement. Thus, for a target ductility ratio, $\mu_{i},\left.R_{\mu}\right|_{\mu=\mu_{i}}$ is defined as

$$
\left.R_{\mu}\right|_{\mu=\mu_{i}}=F_{y, 1} / F_{y, \mu_{i}},
$$

where, $F_{y, 1}$ is the minimum strength required for the oscillator to remain within its linearly elastic limit during the ground motion (i.e., when $\mu=1$ ), and $F_{y, \mu_{i}}$ is the minimum strength at the first yield, for inelastic deformations limited to the ductility ratio of $\mu=\mu_{i} . R_{\mu}(T)$ is a function of the type of nonlinearity in the oscillator for a given damping and ductility ratio of the SDOF oscillator, and earthquake ground motion. An elasto-plastic oscillator is used in this study. The damping is assumed to be $F$-damping (i.e., with no effect of nonlinear behaviour) with value equal to $5 \%$ of critical damping.

For calculating $R_{\mu}(T)$ for a given ground motion, ductility ratio $(\mu)=\mu_{i}$, and initial time period $(T)$, the oscillator has been subjected to the ground motion, and the lateral yield strength, $F_{y, \mu_{i}}$, has been iterated until the calculated displacement ductlity (demand) ratio is within $1 \%$ of $\mu_{i}$. Fourth-order Runge-Kutta method with an adaptive step size control scheme (with maximum step size, $\Delta t=0.004 \mathrm{~s}$ ) has been used to carry out the nonlinear time-history analysis. During the iterations, if more than one values of $F_{y, \mu_{i}}$ are obtained for the same ductility ratio, the largest value has been considered for obtaining the minimum value of $\left.R_{\mu}\right|_{\mu=\mu_{i}}$ which is relevant in design.

In order to study the effects of different ground motion parameters on the SRF spectrum, different ensembles of (synthetic) accelerograms compatible with different PSA spectra have been obtained. The wavelet-based procedure of Mukherjee \& Gupta (2002a) has been used to generate the synthetic accelerograms. In this procedure, a recorded accelerogram is modified to become compatible with a desirable PSA spectrum such that the nonstationary characteristics of the parent accelerogram are preserved. This procedure is generalized enough to modify any accelerogram, irrespective of how much the distributions of energy in the parent accelerogram and the target PSA spectrum differ. The synthetic accelerograms obtained through this procedure have been passed through a hipass filter and then baseline correction has been applied to the filtered accelerograms, in order to obtain realistic estimates of the nonlinear response. Whereas the filtering operation is required to remove the low-frequency corrupting noise from the generated accelerograms, the baseline correction ensures realistic ground velocity and displacement time histories on integration from the corrected accelerograms. The hipass filter used here is Ormsby filter, with the cutoff central frequency of the transition band as $0.06 \mathrm{~Hz}$ and the width of the transition band as $0.02 \mathrm{~Hz}$, and quadratic type baseline correction has been used. The corrected synthetic accelerograms have been used to 
get SRF spectra for the following 56 initial time periods: $0 \cdot 1,0 \cdot 11,0 \cdot 12,0 \cdot 13,0 \cdot 14,0 \cdot 15$, $0 \cdot 16,0 \cdot 17,0 \cdot 18,0 \cdot 19,0 \cdot 20,0 \cdot 22,0 \cdot 24,0 \cdot 26,0 \cdot 28,0 \cdot 30,0 \cdot 32,0 \cdot 34,0 \cdot 36,0 \cdot 38,0 \cdot 40,0 \cdot 42$, $0.44,0.46,0.48,0.50,0.55,0.60,0.65,0.70,0.75,0.80,0 \cdot 85,0.90,0.95,1 \cdot 0,1 \cdot 1,1 \cdot 2,1 \cdot 3$, $1 \cdot 4,1 \cdot 5,1 \cdot 6,1 \cdot 7,1 \cdot 8,1 \cdot 9,2 \cdot 0,2 \cdot 2,2 \cdot 4,2 \cdot 6,2 \cdot 8,3 \cdot 0,3 \cdot 2,3 \cdot 4,3 \cdot 6,3 \cdot 8,4 \cdot 0 \mathrm{~s}$.

The recorded accelerograms used to obtain the ensembles of synthetic accelerograms are from a database of 1274 accelerograms that includes (i) 956 accelerograms recorded during the 106 earthquake events in the western USA region from 1931 to 1984 (see Lee \& Trifunac 1987, for further details), and (ii) 318 accelerograms recorded during the 1994 Northridge earthquake. Even though there is a wide variety in the former set of accelerograms with respect to the source and site characteristics, the latter set of Northridge earthquake accelerograms has been included in order to provide a balanced representation to the near-source ground motions.

The target spectra $P S A(T)$ considered here to examine the parametric dependence of SRF spectra are obtained from the 50\%-confidence level, 5\% damping pseudo spectral velocity (PSV) spectra proposed by Trifunac \& Lee (1985) for the western USA region. These spectra have parameteric dependence on earthquake magnitude, epicentral distance, site conditions, and on focal depth, and are thus deemed to be adequate for the proposed parametric study. Those however do not consider the strong motion duration as a parameter. In view of this and the fact that all the recorded accelerograms considered in this study have different durations, parametric dependence on strong motion duration is studied first.

\section{Parametric dependence on strong motion duration}

Synthetic accelerograms obtained from different recorded accelerograms but compatible with the same PSA spectrum have widely different nonstationary characteristics and strong motion durations. Therefore, it is desirable to examine first how strong motion duration, $T_{s}$, as a parameter affects the SRF spectrum. Previous parametric studies were based on the TrifunacBrady definition (Trifunac \& Brady 1975) for strong motion duration. However, this definition is based on fixed percentages of energy arrival before and during the strong motion phase, and therefore does not always give realistic estimates of the strong motion duration (Bommer \& Martínez-Pereira 1999). In view of this, one generalization of the Trifunac-Brady definition, proposed by Karmakar (2004) and explained in appendix A, is considered for the parametric study here.

In order to study the dependence of SRF spectra on $T_{s}, 1274$ synthetic accelerograms have been generated (from the 1274 recorded accelerograms) such that those are compatible with the PSA spectrum for earthquake magnitude, $M=6 \cdot 5$, epicentral distance, $R=50 \mathrm{~km}$, focal depth, $H=5 \mathrm{~km}$, and alluvium site conditions. Strong motion duration has been calculated for each of these accelerograms, and then, 60 accelerograms for each of the four $T_{s}$ intervals, i.e. 9.44-10.68 s, 18.98-20.88 s, 29.10-31.12 s, and 38.20-41.60 s, corresponding respectively to the central $T_{s}$ values of $10,20,30$ and $40 \mathrm{~s}$, have been identified. SRF spectra for these accelerograms have been calculated in the case of elasto-plastic oscillators with $\mu=4$, and then, mean $R_{\mu}(T)$ spectrum has been obtained for each of the four groups.

Figure 1 shows the comparison of the mean $R_{\mu}(T)$ spectra for the central $T_{s}$ values of 10 , 20,30 and 40 s respectively. Even though duration seems to make some difference to the SRF spectra in this figure, this difference is nominal and devoid of any clear trend. All four curves seem to crisscross one another randomly within a narrow band of SRF values. In view of this, the dependence of SRF spectra on strong motion duration may be ignored in case of the elasto-plastic oscillators. 


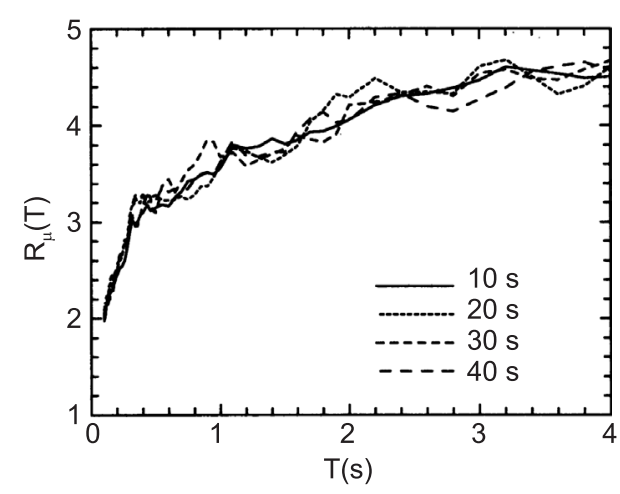

Figure 1. Mean $R_{\mu}(T)$ spectra for different central strong motion durations in case of $\mu=4$.

\section{Parametric dependence on other parameters}

In this section, we study how SRF spectra depend on other parameters like earthquake magnitude, site conditions and epicentral distance. This will be done without giving any weightage to the strong motion duration of the accelerograms, and thus, by treating all synthetic accelerograms alike, irrespective of their strong motion durations. Thus, the uncertainties in the estimation of the SRF spectra would also include the uncertainties, howsoever small those are, due to different strong motion durations, besides the uncertainties due to different nonstationary characteristics of the accelerograms.

\subsection{Data selection and SRF spectrum model}

As mentioned earlier, the parametric study will be carried out by generating synthetic accelerograms for several spectral shapes corresponding to different sets of governing parameters. In order to reduce the computational effort in each of these runs, a smaller subset of the database consisting of 474 recorded accelerograms has been chosen from 72 events (after merging the aftershock event records with the corresponding main event records). This has been done in such a manner that there is no bias in the chosen data in terms of different ground motion parameters. Since 54 earthquake events were associated with less than 14 records each, all records from those earthquakes have been considered. In case of the remaining 18 events, 14 time-histories have been chosen for each event in such a manner that all three types of site conditions (alluvium, hard rock, and intermediate) and a wide range of epicentral distances are covered. In order to verify that the chosen 474 accelerograms represent the characteristics of the population of 1274 accelerograms, 'mean $(m)$ ', 'mean + standard deviation $(\sigma)$ ', and ' $m-\sigma$ ' SRF spectra (for elasto-plastic oscillators with $\mu=4$ ) have been compared for the subset and the entire database of synthetic accelerograms. For this purpose, synthetic accelerograms have been generated for the PSA spectra estimated for $M=4 \cdot 5,5 \cdot 5,6 \cdot 5$, and $R=50 \mathrm{~km}, H=5 \mathrm{~km}$, hard rock site conditions. Figure 2 shows the comparisons of mean, mean $+\sigma$, and mean- $\sigma$ spectra in case of $M=6.5$. The solid lines correspond to the estimates based on the entire population while the dashed lines correspond to the subset of this population. This figure and the figures for $M=4.5$ and 5.5 (as in Karmakar 2004) imply that the estimates of SRF spectra based on the chosen 474 accelerograms should be very close to those based on all 1274 accelerograms.

The parametric study is carried out here by choosing different PSA spectra for various combinations of the governing parameters and by obtaining the SRF spectra at various confidence 


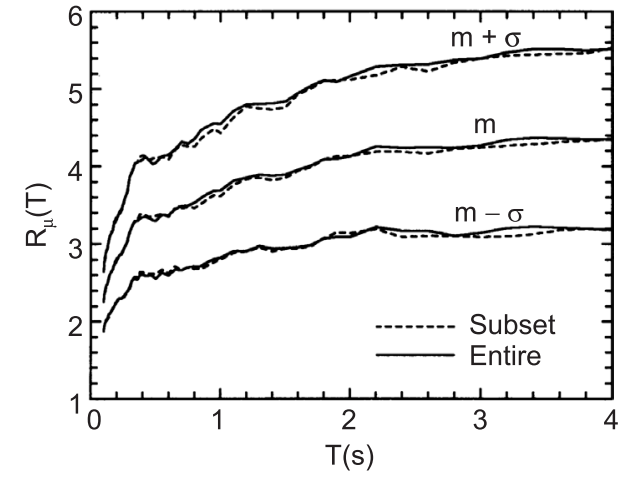

Figure 2. Comparison between $(m-\sigma, m$ and $m+$ $\sigma$ levels) SRF spectra for the subset and the entire database of synthetic accelerograms in case of $M=$ $6 \cdot 5$ and $\mu=4$.

levels such that those spectra account for the uncertainities primarily associated with the nonstationary characteristics of the accelerograms. The parametric study can be conveniently done by directly comparing the SRF spectra (mean or some fixed confidence level) for different PSA spectra involving the desired variation in the parameter under consideration. However, for a compact representation of the results of the parametric study, the SRF spectrum model proposed by Karmakar (2004) is used and parameters of the model are found in case of each PSA spectrum, along with the corresponding error estimates. According to this model, the SRF spectrum for a ground motion may be expressed as

$$
R_{\mu}(T)=\left(\frac{P S A(T)}{P G A}\right)^{\alpha}+\mu\left(1-e^{-\beta T}\right),
$$

where, $P S A(T)$ is the (pseudo spectral acceleration) response spectrum and $P G A$ is the peak ground acceleration of the ground motion. Further, $\alpha$ and $\beta$ are the coefficients determined for a ductility (demand) ratio, $\mu$ and ensemble of ground motions, such that the total squared error, $e$, defined as

$$
e=\sum_{i=1}^{N} \sum_{j=1}^{M}\left(R_{\mu, i j}-\widehat{R}_{\mu, i j}\right)^{2}
$$

is minimized. Here $N(=474)$ is the size of the ensemble, and $M(=56)$ is the number of (initial) time-periods of the elasto-plastic oscillator for which the actual $R_{\mu}(T)$ values have been computed. $R_{\mu, i j}$ and $\widehat{R}_{\mu, i j}$ respectively represent the actual and estimated values of $R_{\mu}$ for the $i$ th sample of the ensemble and the $j$ th time-period.

The $\alpha$ and $\beta$ values (giving the least value of $e$ in (3)) have been computed for 21 combinations of seven $P S A(T)$ curves and $\mu=2,4,6$. Three out of the seven $P S A(T)$ curves (spectrum I, spectrum II, and spectrum III respectively) correspond to $M=4 \cdot 5,5.5$ and 6.5 , with $R=50 \mathrm{~km}, H=5 \mathrm{~km}$, hard rock site conditions. Two curves (spectrum IV and spectrum $\mathrm{V}$ ) correspond to alluvium and intermediate site conditions respectively, with $R=50 \mathrm{~km}, H=5 \mathrm{~km}$, and $M=6.5$, while the remaining two (spectrum VI and spectrum VII) correspond to $R=75$ and $100 \mathrm{~km}$ respectively, with $M=6.5 \mathrm{~km}, H=5 \mathrm{~km}$, hard rock site conditions.

For a compact representation of the error estimates for a set of $P S A(T)$ and $\mu$, error residuals associated with the estimation of SRF spectra (with the help of (2) and computed $\alpha$ 
and $\beta$ parameters) have been estimated as

$$
\epsilon(T)=\log _{10} R_{\mu}(T)-\log _{10} \widehat{R}_{\mu}(T),
$$

for each of the (actual) 474 SRF spectra at 56 values of time-period, $T$. Here, $R_{\mu}(T)$ and $\widehat{R}_{\mu}(T)$ respectively are the actual and estimated values of the SRF spectra at time-period, $T$. Assuming that the residuals follow the normal distribution with mean, $\mu(T)$, and standard deviation, $\sigma(T)$, with

$$
p(\epsilon(T))=\frac{1}{\sigma(T) \sqrt{2 \pi}} \int_{-\infty}^{\epsilon(T)} \exp \left[-\frac{1}{2}\left(\frac{x-\mu(T)}{\sigma(T)}\right)^{2}\right] \mathrm{d} x
$$

describing the probability of confidence associated with $\epsilon(T)$, the values of $\mu(T)=\widehat{\mu}(T)$ and $\sigma(T)=\widehat{\sigma}(T)$ have been calculated from the residuals and smoothened along $T$. This has been done for each combination of $P S A(T)$ and $\mu$. The values, $\widehat{\mu}(T)$ and $\widehat{\sigma}(T)$, may be used to estimate the residual, $\epsilon(T)$, at period $T$ (by using (5)) corresponding to the level of confidence, $p$. This may be then added to $\log _{10} \widehat{R}_{\mu}(T)$ (estimated from (2) with the help of the computed $\alpha$ and $\beta$ values) in order to get the predicted value of $R_{\mu}(T)$ for the confidence level, $p$. The assumption that the residuals are normally distributed has been verified by Karmakar (2004) via the chi-square and Kolmogorov-Smirnov tests.

\subsection{Results and discussion}

As mentioned above, the parametric study is carried out with the help of $\alpha$ and $\beta$ values estimated for 21 combinations of PSA spectra and ductility ratios. Also, error estimates in SRF spectra associated with each set of $\alpha$ and $\beta$ have been obtained with the assumption that those are log-normally distributed.

Table 1 shows the values of $\alpha$ and $\beta$ for all 21 combinations of seven $P S A(T)$ spectra (spectra I, II, III, IV, V, VI and VII) and three $\mu$ values $(=2,4$ and 6$)$. Tables $2-8$ give the corresponding values of $\widehat{\mu}(T)$ and $\widehat{\sigma}(T)$ at 15 selected time periods, with each table showing the results for a particular PSA spectrum. These values may be used along with an interpolation scheme to estimate any intermediate value in the period range of $0 \cdot 1-4 \cdot 0 \mathrm{~s}$.

Figures 3-5 show the comparisons of probabilistic estimates of $R_{\mu}(T)$ corresponding to $\mu=4$ for spectra I, II and III respectively. Each figure shows two sets of such estimates,

Table 1. Coefficients $\alpha$ and $\beta$ of SRF spectrum model for different elastic PSA spectra and

\begin{tabular}{|c|c|c|c|c|c|c|}
\hline \multirow{2}{*}{$\begin{array}{l}\text { PSA } \\
\text { spectrum }\end{array}$} & \multicolumn{2}{|c|}{$\mu=2$} & \multicolumn{2}{|c|}{$\mu=4$} & \multicolumn{2}{|c|}{$\mu=6$} \\
\hline & $\alpha$ & $\beta$ & $\alpha$ & $\beta$ & $\alpha$ & $\beta$ \\
\hline Spectrum I & 0.5640 & $1 \cdot 1072$ & 0.9060 & 1.7088 & $1 \cdot 1400$ & $1 \cdot 5584$ \\
\hline Spectrum II & $0 \cdot 6120$ & $0 \cdot 9192$ & $0 \cdot 9840$ & $1 \cdot 3704$ & 1.2360 & 1.2576 \\
\hline Spectrum III & 0.7980 & 1.0696 & $1 \cdot 2300$ & 1.4832 & $1 \cdot 5540$ & $1 \cdot 3704$ \\
\hline Spectrum IV & 0.7440 & 0.6560 & $1 \cdot 1040$ & 0.9568 & 1.2720 & 0.9568 \\
\hline Spectrum V & 0.7860 & $0 \cdot 8440$ & $1 \cdot 1700$ & $1 \cdot 2200$ & 1.4400 & $1 \cdot 1448$ \\
\hline Spectrum VI & 0.5760 & $0 \cdot 6184$ & $0 \cdot 8460$ & 1.0320 & 0.9960 & 1.0320 \\
\hline Spectrum VII & 0.4620 & 0.5056 & 0.6840 & $0 \cdot 8816$ & $0 \cdot 8160$ & $0 \cdot 8816$ \\
\hline
\end{tabular}
ductility demands. 
Table 2. Estimates of normal distribution parameters for errors in $\log _{10} R_{\mu}(T)$ in case of spectrum I.

\begin{tabular}{|c|c|c|c|c|c|c|}
\hline \multirow{2}{*}{$\begin{array}{l}\text { Period, } \\
T \text { (s) }\end{array}$} & \multicolumn{2}{|c|}{$\mu=2$} & \multicolumn{2}{|c|}{$\mu=4$} & \multicolumn{2}{|c|}{$\mu=6$} \\
\hline & $100 \widehat{\mu}(T)$ & $10 \widehat{\sigma}(T)$ & $100 \widehat{\mu}(T)$ & $10 \widehat{\sigma}(T)$ & $100 \widehat{\mu}(T)$ & $10 \widehat{\sigma}(T)$ \\
\hline $0 \cdot 10$ & $-5 \cdot 360$ & 0.653 & -7.590 & $0 \cdot 835$ & -9.270 & $0 \cdot 847$ \\
\hline $0 \cdot 15$ & -3.790 & 0.760 & $-4 \cdot 560$ & $0 \cdot 907$ & $-5 \cdot 270$ & 0.936 \\
\hline $0 \cdot 20$ & $-2 \cdot 100$ & 0.784 & -1.570 & $0 \cdot 940$ & $-1 \cdot 060$ & 1.003 \\
\hline $0 \cdot 30$ & 0.450 & 0.830 & 1.710 & 1.038 & 4.050 & 1.094 \\
\hline 0.40 & $2 \cdot 440$ & $0 \cdot 813$ & 3.680 & $1 \cdot 010$ & $6 \cdot 140$ & 1.023 \\
\hline $0 \cdot 50$ & $2 \cdot 710$ & 0.806 & $2 \cdot 660$ & $1 \cdot 013$ & $4 \cdot 370$ & 1.025 \\
\hline $0 \cdot 60$ & $2 \cdot 300$ & 0.789 & 1.480 & 1.003 & 2.750 & 1.031 \\
\hline 0.70 & 1.890 & 0.783 & $0 \cdot 260$ & 0.987 & $1 \cdot 220$ & 1.026 \\
\hline $0 \cdot 80$ & 1.080 & 0.793 & $-1 \cdot 380$ & 0.993 & -0.830 & $1 \cdot 031$ \\
\hline $0 \cdot 90$ & $0 \cdot 170$ & $0 \cdot 817$ & $-2 \cdot 940$ & $0 \cdot 996$ & -2.940 & 1.028 \\
\hline 1.00 & -0.580 & 0.830 & $-3 \cdot 720$ & 0.987 & $-4 \cdot 270$ & $1 \cdot 010$ \\
\hline $1 \cdot 50$ & -2.650 & 0.780 & $-6 \cdot 510$ & $0 \cdot 965$ & -9.060 & 0.969 \\
\hline $2 \cdot 00$ & $-4 \cdot 380$ & 0.780 & -7.730 & 0.942 & $-11 \cdot 180$ & 0.966 \\
\hline $3 \cdot 00$ & -5.900 & 0.761 & -8.790 & 0.920 & $-12 \cdot 510$ & 0.972 \\
\hline $4 \cdot 00$ & $-5 \cdot 390$ & 0.758 & $-6 \cdot 860$ & $0 \cdot 985$ & $-10 \cdot 400$ & 1.053 \\
\hline
\end{tabular}

with each set showing the SRF estimates for $p=0 \cdot 1,0 \cdot 5$, and $0 \cdot 9$. The two sets correspond to (i) the actual SRF data for 474 synthetic accelerograms (see the dashed curves), and (ii) the SRF data estimated by using the model of (2) along with the $\alpha$ and $\beta$ values from table 1 and $\widehat{\mu}(T)$ and $\widehat{\sigma}(T)$ values from tables $2-4$ (see the solid curves). It may be observed that there is excellent agreement between the estimated and actual SRF curves for all three confidence levels in each case, and thus, there is no additional uncertainty introduced into the SRF estimates due to using the model of (2). The $80 \%$ confidence interval bounded by the two

Table 3. Estimates of normal distribution parameters for errors in $\log _{10} R_{\mu}(T)$ in case of spectrum II.

\begin{tabular}{|c|c|c|c|c|c|c|}
\hline \multirow{2}{*}{$\begin{array}{l}\text { Period, } \\
T(\mathrm{~s})\end{array}$} & \multicolumn{2}{|c|}{$\mu=2$} & \multicolumn{2}{|c|}{$\mu=4$} & \multicolumn{2}{|c|}{$\mu=6$} \\
\hline & $100 \widehat{\mu}(T)$ & $10 \widehat{\sigma}(T)$ & $100 \widehat{\mu}(T)$ & $10 \widehat{\sigma}(T)$ & $100 \widehat{\mu}(T)$ & $10 \widehat{\sigma}(T)$ \\
\hline $0 \cdot 10$ & $-3 \cdot 130$ & 0.589 & -4.910 & 0.754 & $-9 \cdot 810$ & 0.794 \\
\hline $0 \cdot 15$ & $-3 \cdot 000$ & 0.690 & -3.930 & 0.858 & $-8 \cdot 110$ & 0.888 \\
\hline $0 \cdot 20$ & $-2 \cdot 100$ & 0.717 & $-2 \cdot 240$ & 0.877 & $-5 \cdot 310$ & 0.912 \\
\hline $0 \cdot 30$ & $-0 \cdot 380$ & 0.774 & 0.520 & 0.981 & -0.090 & 0.988 \\
\hline 0.40 & 1.640 & 0.794 & 2.900 & $0 \cdot 961$ & $3 \cdot 570$ & 0.978 \\
\hline 0.50 & $2 \cdot 060$ & 0.783 & $2 \cdot 370$ & 0.973 & $3 \cdot 280$ & 0.977 \\
\hline $0 \cdot 60$ & 1.810 & 0.782 & 1.430 & 0.971 & $2 \cdot 440$ & 0.991 \\
\hline $0 \cdot 70$ & 1.510 & 0.782 & 0.340 & 0.970 & 1.450 & 0.998 \\
\hline $0 \cdot 80$ & 0.990 & 0.784 & -0.980 & 0.984 & $0 \cdot 140$ & 1.005 \\
\hline 0.90 & 0.440 & 0.802 & -1.900 & 0.997 & -1.040 & $1 \cdot 011$ \\
\hline 1.00 & -0.020 & 0.819 & $-2 \cdot 220$ & 0.995 & -1.710 & 1.009 \\
\hline 1.50 & -1.620 & 0.775 & -3.950 & 0.989 & $-4 \cdot 610$ & 1.036 \\
\hline $2 \cdot 00$ & -2.740 & 0.834 & $-4 \cdot 200$ & 0.997 & $-5 \cdot 660$ & 1.051 \\
\hline $3 \cdot 00$ & $-4 \cdot 130$ & 0.812 & -4.470 & 1.020 & $-6 \cdot 230$ & 1.079 \\
\hline $4 \cdot 00$ & $-3 \cdot 870$ & 0.804 & $-2 \cdot 520$ & 1.067 & -3.980 & $1 \cdot 171$ \\
\hline
\end{tabular}


Table 4. Estimates of normal distribution parameters for errors in $\log _{10} R_{\mu}(T)$ in case of spectrum III.

\begin{tabular}{|c|c|c|c|c|c|c|}
\hline \multirow{2}{*}{$\begin{array}{l}\text { Period, } \\
T(\mathrm{~s})\end{array}$} & \multicolumn{2}{|c|}{$\mu=2$} & \multicolumn{2}{|c|}{$\mu=4$} & \multicolumn{2}{|c|}{$\mu=6$} \\
\hline & $100 \widehat{\mu}(T)$ & $10 \widehat{\sigma}(T)$ & $100 \widehat{\mu}(T)$ & $10 \widehat{\sigma}(T)$ & $100 \widehat{\mu}(T)$ & $10 \widehat{\sigma}(T)$ \\
\hline $0 \cdot 10$ & -0.460 & $0 \cdot 552$ & -0.610 & 0.725 & -1.780 & 0.756 \\
\hline $0 \cdot 15$ & $-1 \cdot 540$ & 0.635 & -1.430 & $0 \cdot 816$ & $-2 \cdot 370$ & $0 \cdot 845$ \\
\hline $0 \cdot 20$ & -1.470 & 0.663 & $-1 \cdot 260$ & $0 \cdot 825$ & $-1 \cdot 820$ & $0 \cdot 850$ \\
\hline $0 \cdot 30$ & -1.070 & 0.734 & -0.430 & 0.929 & 0.290 & 0.946 \\
\hline 0.40 & 0.410 & 0.770 & $1 \cdot 200$ & 0.945 & 1.770 & 0.953 \\
\hline $0 \cdot 50$ & $0 \cdot 550$ & $0 \cdot 761$ & 0.460 & $0 \cdot 942$ & $0 \cdot 820$ & $0 \cdot 951$ \\
\hline $0 \cdot 60$ & $0 \cdot 140$ & 0.775 & $-0 \cdot 540$ & 0.943 & $-0 \cdot 240$ & 0.973 \\
\hline $0 \cdot 70$ & -0.180 & 0.785 & -1.430 & 0.954 & -1.090 & 1.000 \\
\hline $0 \cdot 80$ & -0.570 & 0.793 & $-2 \cdot 190$ & 0.976 & $-1 \cdot 830$ & 1.021 \\
\hline $0 \cdot 90$ & -0.890 & $0 \cdot 808$ & $-2 \cdot 470$ & 0.994 & $-2 \cdot 190$ & 1.029 \\
\hline $1 \cdot 00$ & -1.050 & 0.821 & $-2 \cdot 380$ & 0.993 & $-2 \cdot 160$ & 1.033 \\
\hline $1 \cdot 50$ & $-1 \cdot 540$ & 0.797 & $-2 \cdot 300$ & 1.019 & $-2 \cdot 600$ & $1 \cdot 102$ \\
\hline $2 \cdot 00$ & -1.940 & $0 \cdot 840$ & -0.890 & $1 \cdot 053$ & $-1 \cdot 870$ & $1 \cdot 131$ \\
\hline $3 \cdot 00$ & -1.780 & 0.838 & $-0 \cdot 220$ & $1 \cdot 122$ & $-1 \cdot 260$ & $1 \cdot 198$ \\
\hline $4 \cdot 00$ & $-1 \cdot 140$ & $0 \cdot 850$ & $1 \cdot 170$ & $1 \cdot 143$ & -0.040 & $1 \cdot 242$ \\
\hline
\end{tabular}

extreme solid or dashed lines in each figure represents the high level of uncertainty associated with using an arbitrary accelerogram in the method of Mukherjee \& Gupta (2002b), and this is due to a large variety of accelerograms with very different nonstationary characteristics used in this study. It may be mentioned that the coefficient of variation is between 0.20 and 0.25 in the period range of $0.2-0.8 \mathrm{~s}$ which is larger than that obtained by Mukherjee \& Gupta (2002b) on the basis of 35 recorded accelerograms of $20 \mathrm{~s}$ strong motion duration. It appears unlikely that a real-life situation would encounter higher levels of uncertainty due to the lack

Table 5. Estimates of normal distribution parameters for errors in $\log _{10} R_{\mu}(T)$ in case of spectrum IV.

\begin{tabular}{|c|c|c|c|c|c|c|}
\hline \multirow{2}{*}{$\begin{array}{l}\text { Period, } \\
T \text { (s) }\end{array}$} & \multicolumn{2}{|c|}{$\mu=2$} & \multicolumn{2}{|c|}{$\mu=4$} & \multicolumn{2}{|c|}{$\mu=6$} \\
\hline & $100 \widehat{\mu}(T)$ & $10 \widehat{\sigma}(T)$ & $100 \widehat{\mu}(T)$ & $10 \widehat{\sigma}(T)$ & $100 \widehat{\mu}(T)$ & $10 \widehat{\sigma}(T)$ \\
\hline 0.10 & 1.840 & 0.448 & 2.180 & 0.626 & 1.510 & 0.666 \\
\hline 0.15 & 0.060 & 0.533 & 0.120 & 0.712 & -0.480 & 0.748 \\
\hline 0.20 & -0.760 & 0.583 & -0.990 & 0.739 & -1.340 & 0.768 \\
\hline 0.30 & -1.630 & 0.681 & -1.320 & 0.852 & -0.880 & 0.882 \\
\hline 0.40 & -0.570 & 0.734 & 0.160 & 0.889 & 0.150 & 0.907 \\
\hline 0.50 & -0.310 & 0.733 & -0.130 & 0.896 & -0.570 & 0.904 \\
\hline 0.60 & -0.410 & 0.743 & -0.840 & 0.907 & -1.340 & 0.930 \\
\hline 0.70 & -0.330 & 0.756 & -1.320 & 0.928 & -1.950 & 0.957 \\
\hline 0.80 & -0.370 & 0.771 & -1.690 & 0.957 & -2.320 & 0.979 \\
\hline 0.90 & -0.450 & 0.786 & -1.760 & 0.978 & -2.290 & 0.992 \\
\hline 1.00 & -0.430 & 0.792 & -1.610 & 0.979 & -2.120 & 1.001 \\
\hline 1.50 & -0.400 & 0.793 & -1.840 & 0.989 & -2.550 & 1.067 \\
\hline 2.00 & -0.940 & 0.837 & -0.910 & 1.029 & -1.970 & 1.105 \\
\hline 3.00 & -1.480 & 0.842 & -0.110 & 1.121 & -0.600 & 1.200 \\
\hline 4.00 & -1.360 & 0.840 & 1.860 & 1.161 & 1.430 & 1.251 \\
\hline
\end{tabular}


Table 6. Estimates of normal distribution parameters for errors in $\log _{10} R_{\mu}(T)$ in case of spectrum V.

\begin{tabular}{|c|c|c|c|c|c|c|}
\hline \multirow{2}{*}{$\begin{array}{l}\text { Period, } \\
T \text { (s) }\end{array}$} & \multicolumn{2}{|c|}{$\mu=2$} & \multicolumn{2}{|c|}{$\mu=4$} & \multicolumn{2}{|c|}{$\mu=6$} \\
\hline & $100 \widehat{\mu}(T)$ & $10 \widehat{\sigma}(T)$ & $100 \widehat{\mu}(T)$ & $10 \widehat{\sigma}(T)$ & $100 \widehat{\mu}(T)$ & $10 \widehat{\sigma}(T)$ \\
\hline 0.10 & 0.660 & 0.507 & 0.890 & 0.677 & 0.020 & 0.726 \\
\hline 0.15 & -0.900 & 0.586 & -0.750 & 0.763 & -1.620 & 0.796 \\
\hline 0.20 & -1.340 & 0.620 & -1.300 & 0.783 & -1.870 & 0.805 \\
\hline 0.30 & -1.470 & 0.712 & -1.110 & 0.895 & -0.590 & 0.917 \\
\hline 0.40 & -0.020 & 0.759 & 0.490 & 0.917 & 0.740 & 0.928 \\
\hline 0.50 & 0.230 & 0.752 & -0.040 & 0.918 & 0.130 & 0.927 \\
\hline 0.60 & -0.030 & 0.757 & -0.930 & 0.923 & -0.760 & 0.952 \\
\hline 0.70 & -0.140 & 0.767 & -1.640 & 0.941 & -1.460 & 0.978 \\
\hline 0.80 & -0.310 & 0.783 & -2.230 & 0.968 & -1.980 & 1.001 \\
\hline 0.90 & -0.500 & 0.799 & -2.420 & 0.988 & -2.110 & 1.014 \\
\hline 1.00 & -0.590 & 0.806 & -2.300 & 0.988 & -1.990 & 1.021 \\
\hline 1.50 & -0.940 & 0.790 & -2.410 & 1.007 & -2.530 & 1.082 \\
\hline 2.00 & -1.490 & 0.840 & -1.210 & 1.041 & -1.880 & 1.120 \\
\hline 3.00 & -1.760 & 0.837 & -0.340 & 1.124 & -0.920 & 1.203 \\
\hline 4.00 & -1.230 & 0.842 & 1.490 & 1.156 & 0.870 & 1.256 \\
\hline
\end{tabular}

of knowledge about the nonstationary characteristics of the design ground motion. One may actually consider lower levels of uncertainty if the past records at the site of interest or in its neighbourhood are available. It may be noteworthy that the coefficient of variation obtained by Tiwari \& Gupta (2000) in predicting SRF spectra in terms of various governing parameters ranged between 0.4 and 0.5 which is much higher than the present level of uncertainty. This is so as their model also accounted for the uncertainty associated with the specification of the PSA spectrum.

Table 7. Estimates of normal distribution parameters for errors in $\log _{10} R_{\mu}(T)$ in case of spectrum VI.

\begin{tabular}{|c|c|c|c|c|c|c|}
\hline \multirow{2}{*}{$\begin{array}{l}\text { Period, } \\
T(\mathrm{~s})\end{array}$} & \multicolumn{2}{|c|}{$\mu=2$} & \multicolumn{2}{|c|}{$\mu=4$} & \multicolumn{2}{|c|}{$\mu=6$} \\
\hline & $100 \widehat{\mu}(T)$ & $10 \widehat{\sigma}(T)$ & $100 \widehat{\mu}(T)$ & $10 \widehat{\sigma}(T)$ & $100 \widehat{\mu}(T)$ & $10 \widehat{\sigma}(T)$ \\
\hline 0.10 & -0.870 & 0.490 & -1.400 & 0.653 & -2.340 & 0.699 \\
\hline 0.15 & -1.770 & 0.586 & -1.900 & 0.760 & -2.540 & 0.794 \\
\hline 0.20 & -1.840 & 0.627 & -1.860 & 0.790 & -2.210 & 0.814 \\
\hline 0.30 & -1.300 & 0.723 & -0.910 & 0.906 & -0.240 & 0.928 \\
\hline 0.40 & 0.410 & 0.769 & 0.960 & 0.935 & 1.070 & 0.941 \\
\hline 0.50 & 0.760 & 0.761 & 0.370 & 0.932 & 0.220 & 0.943 \\
\hline 0.60 & 0.670 & 0.772 & -0.440 & 0.935 & -0.620 & 0.967 \\
\hline 0.70 & 0.670 & 0.781 & -1.120 & 0.948 & -1.230 & 0.995 \\
\hline 0.80 & 0.590 & 0.791 & -1.690 & 0.973 & -1.740 & 1.016 \\
\hline 0.90 & 0.510 & 0.807 & -1.860 & 0.995 & -1.890 & 1.026 \\
\hline 1.00 & 0.520 & 0.819 & -1.760 & 0.993 & -1.760 & 1.031 \\
\hline 1.50 & 0.100 & 0.794 & -2.170 & 1.018 & -2.270 & 1.102 \\
\hline 2.00 & -0.860 & 0.841 & -1.420 & 1.053 & -1.950 & 1.134 \\
\hline 3.00 & -2.230 & 0.837 & -1.580 & 1.125 & -1.930 & 1.199 \\
\hline 4.00 & -2.780 & 0.849 & -0.470 & 1.145 & -0.970 & 1.242 \\
\hline
\end{tabular}


Table 8. Estimates of normal distribution parameters for errors in $\log _{10} R_{\mu}(T)$ in case of spectrum VII.

\begin{tabular}{|c|c|c|c|c|c|c|}
\hline \multirow{2}{*}{$\begin{array}{l}\text { Period, } \\
T \text { (s) }\end{array}$} & \multicolumn{2}{|c|}{$\mu=2$} & \multicolumn{2}{|c|}{$\mu=4$} & \multicolumn{2}{|c|}{$\mu=6$} \\
\hline & $100 \widehat{\mu}(T)$ & $10 \widehat{\sigma}(T)$ & $100 \widehat{\mu}(T)$ & $10 \widehat{\sigma}(T)$ & $100 \widehat{\mu}(T)$ & $10 \widehat{\sigma}(T)$ \\
\hline 0.10 & -1.280 & 0.470 & -2.820 & 0.620 & -3.610 & 0.674 \\
\hline 0.15 & -1.900 & 0.560 & -2.490 & 0.733 & -2.850 & 0.769 \\
\hline 0.20 & -2.000 & 0.613 & -2.240 & 0.776 & -2.000 & 0.800 \\
\hline 0.30 & -1.370 & 0.721 & -0.840 & 0.903 & 0.870 & 0.923 \\
\hline 0.40 & 0.290 & 0.769 & 1.230 & 0.935 & 2.650 & 0.941 \\
\hline 0.50 & 0.630 & 0.761 & 0.660 & 0.932 & 2.080 & 0.943 \\
\hline 0.60 & 0.520 & 0.772 & -0.170 & 0.934 & 1.420 & 0.966 \\
\hline 0.70 & 0.530 & 0.780 & -0.850 & 0.947 & 0.910 & 0.994 \\
\hline 0.80 & 0.480 & 0.791 & -1.410 & 0.973 & 0.440 & 1.015 \\
\hline 0.90 & 0.430 & 0.807 & -1.620 & 0.994 & 0.300 & 1.024 \\
\hline 1.00 & 0.460 & 0.819 & -1.550 & 0.990 & 0.440 & 1.027 \\
\hline 1.50 & 0.090 & 0.793 & -2.140 & 1.016 & -0.350 & 1.101 \\
\hline 2.00 & -0.970 & 0.842 & -1.680 & 1.050 & -0.560 & 1.133 \\
\hline 3.00 & -2.830 & 0.837 & -2.350 & 1.125 & -1.520 & 1.198 \\
\hline 4.00 & -3.880 & 0.842 & -1.550 & 1.142 & -1.150 & 1.240 \\
\hline
\end{tabular}
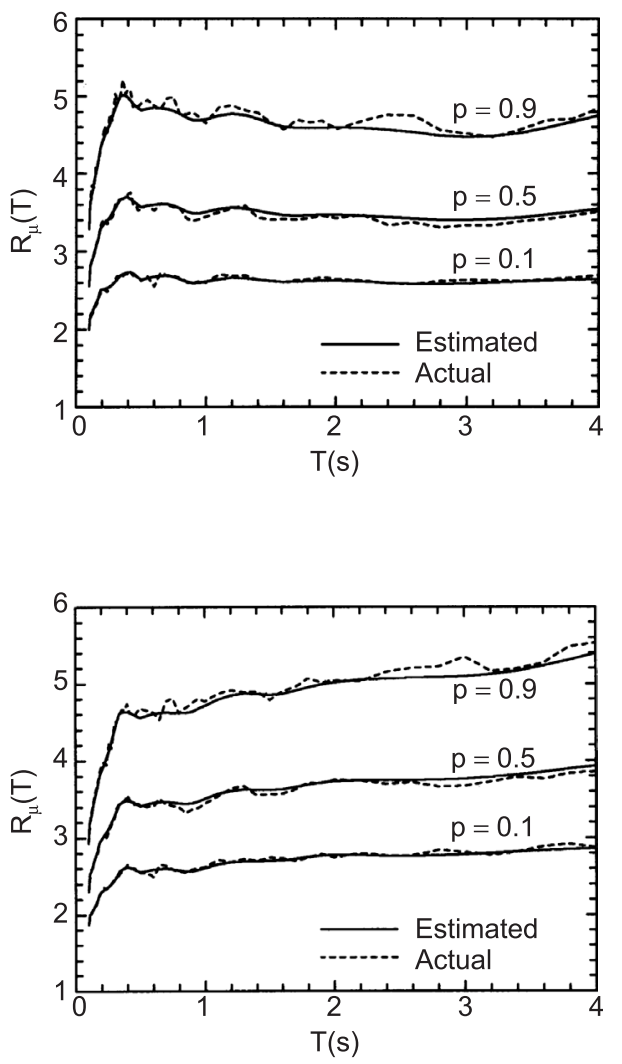

Figure 3. Comparison of actual and estimated SRF spectra for different confidence levels in case of spectrum I and $\mu=4$.

Figure 4. Comparison of actual and estimated SRF spectra for different confidence levels in case of spectrum II and $\mu=4$. 


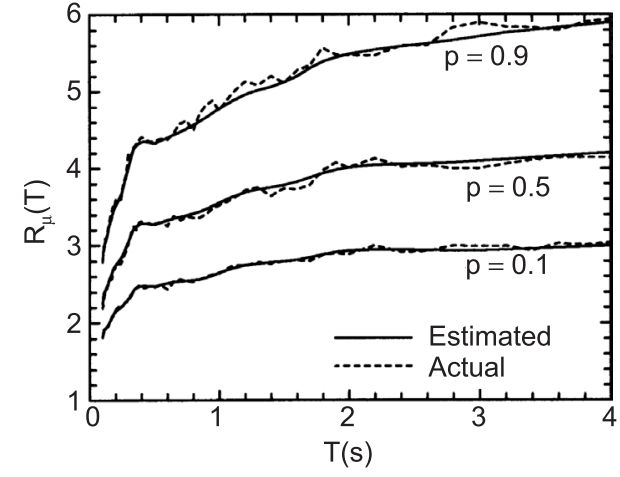

Figure 5. Comparison of actual and estimated SRF spectra for different confidence levels in case of spectrum III and $\mu=4$.

In order to study the dependence of SRF spectrum on earthquake magnitude, the results in the first three rows of table 1 (for spectra I, II and III) are compared. It may be observed that the values of $\alpha$ increase with earthquake magnitude, with a steeper increase in case of higher magnitudes. The values of $\beta$ decrease significantly from $M=4.5$ to 5.5 and then increase moderately from $M=5.5$ to $6 \cdot 5$. Figure 6 shows the comparison of the $R_{\mu}(T)$ spectra obtained for spectra I, II and III by using the $\alpha$ and $\beta$ values for $\mu=4$ and error estimates for $50 \%$ confidence level. It is observed that lower magnitudes give higher values of SRF for short to medium period range $(T<1.0 \mathrm{~s}$ ), while higher magnitudes give higher values of SRF for long period range $(T>1.0 \mathrm{~s})$. The effect of earthquake magnitude is particularly significant for the periods larger than $2 \mathrm{~s}$. These findings are in very good agreement with those reported by Tiwari \& Gupta (2000), and in complete contradiction with those of Miranda (1993).

Comparison of $\alpha$ and $\beta$ values in the 3rd, 4th and 5th rows of table 1 (for spectra III, IV and V) shows that both parameters increase as we go from alluvium site conditions to intermediate site conditions and then to hard rock conditions. A comparison of the $R_{\mu}(T)$ spectra obtained from these values for $\mu=4$ and $p=0.5$ is shown in figure 7 . It may be observed that except for long periods $(T>2.5 \mathrm{~s})$, hard rock conditions are associated with greater SRF values. However, the effect of site conditions is not as strong as was observed in the case of earthquake magnitude. These results are in agreement with those of Chakraborti $\&$ Gupta (2005) for very long periods $(T>3 \mathrm{~s})$, with those of Tiwari \& Gupta (2000) for

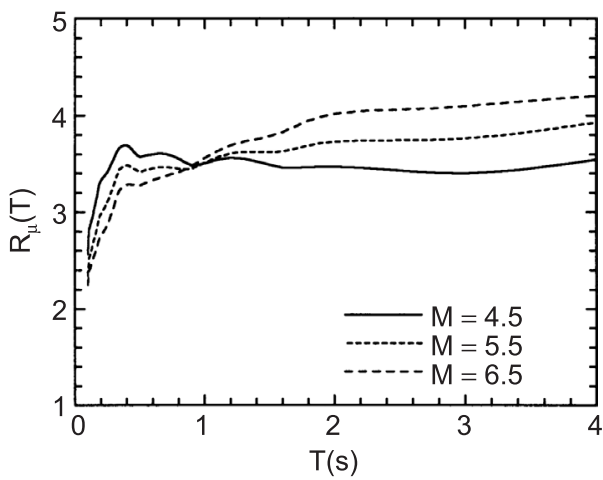

Figure 6. Estimated SRF spectra for different earthquake magnitudes in case of $R=50 \mathrm{~km}, H=5 \mathrm{~km}$, hard rock site, $p=0 \cdot 5$, and $\mu=4$. 


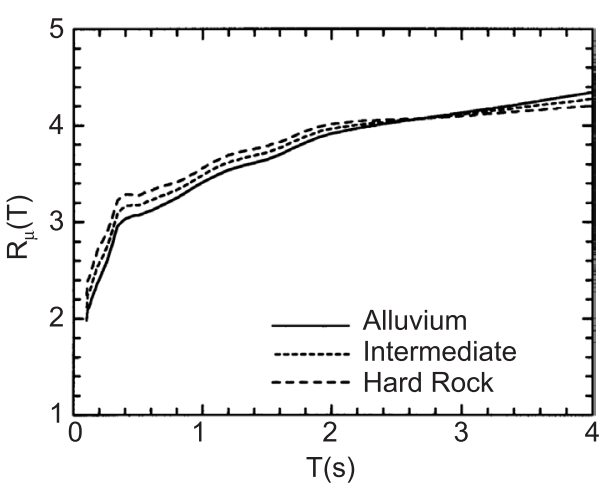

Figure 7. Estimated SRF spectra for different site conditions in case of $R=50 \mathrm{~km}, H=5 \mathrm{~km}, M=$ $6 \cdot 5, p=0 \cdot 5$, and $\mu=4$.

short to medium periods $(T<0.8 \mathrm{~s})$, and with those of Elghadamsi \& Mohraz (1987) for a large range of periods $(T<2.5 \mathrm{~s})$.

The effects of epicentral distance on $\alpha$ and $\beta$ may be seen in table 1 by comparing the $3 \mathrm{rd}$, 6 th and 7 th rows (for spectra III, VI and VII). Both parameters decrease with an increase in the epicentral distance. However, this does not lead to an appreciable effect of epicentral distance on the SRF spectra, as shown by figure 8 in case of $\mu=4$ and $p=0 \cdot 5$. This confirms the observations of Miranda (1993) and Krawinkler \& Nassar (1990) that the effects of epicentral distance on SRF spectrum may be neglected completely.

\section{Conclusions}

A parametric study has been carried out to study the explicit dependence of SRF spectrum on strong motion duration, earthquake magnitude, site conditions, and epicentral distance. This has been done in case of elasto-plastic oscillators without degradations, and with the help of 1274 accelerograms recorded in western USA during 107 earthquake events. The recorded accelerograms have been modified so as to conform to the same PSA spectrum for a particular set of parameters.

It is seen that the dependence of SRF spectra on strong motion duration may be ignored in the case of elasto-plastic oscillators. The parametric study with respect to earthquake magnitude has confirmed the significant dependence of SRF spectrum on this parameter, particularly

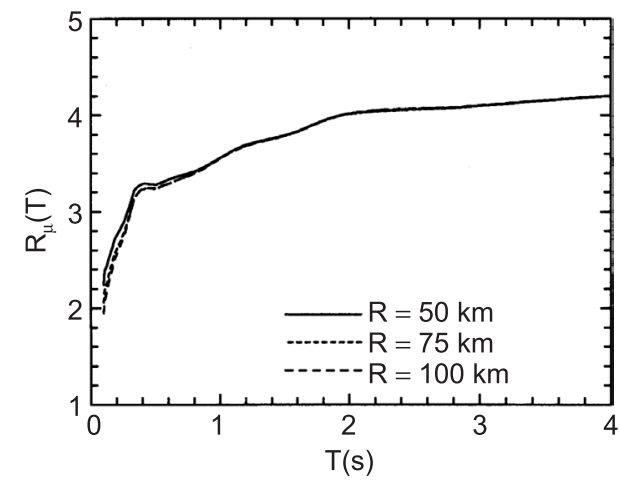

Figure 8. Estimated SRF spectra for different epicentral distances in case of $M=6 \cdot 5, H=5 \mathrm{~km}$, hard rock site, $p=0 \cdot 5$, and $\mu=4$. 
for the periods larger than $2 \mathrm{~s}$. It has been shown that higher magnitude earthquakes are associated with lower values of SRFs in the short to medium period range $(T<1.0 \mathrm{~s})$ and with higher values of SRFs in the long period range $(T>1.0 \mathrm{~s})$. The effect of site conditions however is not found to be as strong. Further, except for very long periods ( $T>2.5 \mathrm{~s})$, hard rock conditions are found to be associated with greater SRF values. There is no appreciable effect of the epicentral distance on the SRF spectra, as was also shown by some of the earlier investigations.

For each set of parameters in the parametric study and three ductility demands, the parameters of the SRF spectrum model proposed by Karmakar (2004) have been estimated along with the log-normally distributed error estimates. Those can be used, if applicable, to predict the SRF spectrum at a given level of confidence, with the uncertainty primarily related to the type of nonstationarity in the ground motion.

\section{Appendix A. Duration definition (Karmakar 2004)}

The definition of strong motion duration by Karmakar (2004) is a 'significant duration' definition (Bommer \& Martínez-Pereira 1999), defined as

$$
T_{s}=T_{2}-T_{1},
$$

where $T_{1}$ and $T_{2}$ respectively are the initial and final cut-off times corresponding to certain percentages of the total energy arrival in the accelerogram. Those correspond to $E_{n}(t)=E_{1}$ and $E_{2}$ respectively, where $E_{n}(t)$ is the normalized energy level at time $t$, expressed as

$$
E_{n}(t)=\left[\int_{0}^{t} x^{2}(t) \mathrm{d} t\right] /\left[\int_{0}^{T} x^{2}(t) \mathrm{d} t\right]
$$

with $x(t)$ denoting the time-history, and $T$ denoting the total duration of the time-history. The strong motion duration by Trifunac $\&$ Brady (1975) is based on the fixed values, $E_{1}=0.05$ and $E_{2}=0.95$.

Karmakar (2004) proposed making $E_{1}$ and $E_{2}$ variable for an accelerogram such that the total energy $\Delta E\left(=E_{2}-E_{1}\right)$ associated with the strong motion phase arrives in the minimum time and that this (maximum) rate of energy arrival is stationary around the chosen value of $\Delta E$. In the first step, several values of $\Delta E$ are considered, and then that value of $T_{s}$ is considered for each of the $\Delta E$ values which minimizes $T_{s}\left(=T_{2}-T_{1}\right)$ by a suitable choice of $E_{1}$. Through this step, the strong motion segment of the accelerogram is identified for a given $\Delta E$. In the second step, the largest value of $\Delta E$, say $(\Delta E)_{\text {stationary }}$, that is associated with a stationary rate of energy arrival with respect to the neighbouring lower $\Delta E$ values is identified. This is done by calculating the average rate of energy arrival i.e. $\Delta E / T_{s}$ at every value of $\Delta E$ with reference to the window, $(\Delta E-0.05, \Delta E)$, and by plotting the average deviation squared with respect to the average value with $\Delta E$. The largest value of $\Delta E$ at which a local minimum occurs is taken as $(\Delta E)_{\text {stationary }}$, and the value of $T_{s}$ corresponding to this value of $\Delta E$ in the first step is taken as the strong motion duration.

\section{References}

ATC 1995 Structural response modification factors. Report ATC-19. Applied Technology Council, Redwood City, CA, USA 
Bommer J J, Martínez-Pereira A 1999 The effective duration of earthquake strong motion. J. Earthquake Eng. 3: 127-172

Chakraborti A, Gupta V K 2005 Scaling of strength reduction factors for degrading elasto-plastic oscillators. Earthquake Eng. Struct. Dyn. 34: 189-206

Elghadamsi F E, Mohraz B 1987 Inelastic earthquake spectra. Earthquake Eng. Struct. Dyn. 15: 91104

Karmakar D 2004 Design spectrum-based scaling of strength reduction factors. M. Tech. Thesis, Dept. of Civil Eng. IIT, Kanpur

Krawinkler H, Nassar A 1990 Strength and ductility demands for SDOF and MDOF systems subjected to Whittier Narrows earthquake ground motions, CSMIP-1990, California Dept. of Conservation, Sacramento, CA, USA

Krawinkler H, Rahnama M 1992 Effects of soil on design spectra. Proc. 10th Wld Conf. Earthq. Eng. Madrid, Spain 10: 5841-5846

Lee V W, Trifunac M D 1987 Strong earthquake ground motion data in EQUINFOS: Part 1. Report No. CE 87-01, Dept. of Civil Eng., Univ. of Southern California, Los Angeles, USA

Miranda E 1993 Site-dependent strength reduction factors. J. Struct. Eng., ASCE 119: 3503-3519

Mukherjee S, Gupta V K 2002a Wavelet-based generation of spectrum-compatible time-histories. Soil Dyn. Earthquake Eng. 22: 799-804

Mukherjee S, Gupta V K 2002b Wavelet-based characterization of design ground motions. Earthquake Eng. Struct. Dyn. 31: 1173-1190

Newmark N M, Hall W J 1973 Procedures and criteria for earthquake resistant design, in building practices for disaster mitigation. Building Science Series 46, National Bureau of Standards, U.S. Dept. of Commerce, Washington, D.C., USA, 1: 209-236

Peng M H, Elghadamsi F, Mohraz B 1988 A stochastic procedure for nonlinear response spectra. Ninth Wld Conf. Earthq. Eng. Tokyo-Kyoto, Japan, 5: 1069-1074

Riddell R, Newmark N M 1979 Statistical analysis of the response of nonlinear systems subjected to earthquakes. Structural Research Series No. 468, Dept. of Civil Eng., Univ. of Illinois, Urbana, IL, USA

Tiwari A K, Gupta V K 2000 Scaling of ductility and damage-based strength reduction factors for horizontal motions. Earthquake Eng. Struct. Dyn. 29: 969-987

Trifunac M D, Brady A G 1975 A study of the duration of strong earthquake ground motion. Bull. Seismol. Soc. Am. 65: 581-626

Trifunac M D, Lee V W 1985 Preliminary empirical models for scaling pseudo relative velocity spectra of strong earthquake accelerations in terms of magnitude, distance, site intensity and recording site conditions, Report No. CE 85-04, Dept. of Civil Eng., Univ. of Southern California, Los Angeles, CA, USA 